\title{
Hippocampal volume and the AKT signaling system in first-episode schizophrenia
}

\author{
András Szamosi ${ }^{a}$, Oguz Kelemen ${ }^{\text {b }}$, Szabolcs Kéri ${ }^{\text {a,c,* }}$ \\ ${ }^{a}$ National Psychiatry Center, Budapest, Hungary \\ b Bács-Kiskun County Hospital, Psychiatry Center, Kecskemét, Hungary \\ ${ }^{\mathrm{c}}$ University of Szeged, Faculty of Medicine, Department of Physiology, Szeged, Hungary
}

\section{A R T I C L E I N F O}

\section{Article history:}

Received 10 November 2011

Received in revised form

9 December 2011

Accepted 12 December 2011

\section{Keywords:}

Schizophrenia

Hippocampus

AKT system

Neuregulin 1

\begin{abstract}
A B S T R A C T
Objective: The phosphoinositide $3^{\prime}$-kinase (PI3K) - protein kinase B (AKT1) - glycogen synthase kinase (GSK)-3 $\beta$ system is modulated by several factors implicated in the pathophysiology of schizophrenia. Evidence suggests that neuregulin 1 (NRG1) induces decreased AKT phosphorylation in schizophrenia relative to healthy controls, which may be related to dysfunctional neurodevelopment and neuroplasticity. The aim of this study was to investigate the relationship between NRG1 - induced AKT phosphorylation and hippocampal volume in schizophrenia.

Methods: Participants were 20 first-episode patients with schizophrenia who did not receive psychotropic medications and 20 matched healthy controls. We measured the phosphorylated AKT - total AKT and phosphorylated ERK (extracellular signal-regulated kinase) - total ERK ratios in peripheral lymphoblasts before and after NRG1 administration. Whole-brain, left, and right hippocampal volumes were quantified using FreeSurfer software.

Results: Patients with schizophrenia displayed decreased AKT but normal ERK ratio compared with controls. Patients also had a reduction in left hippocampal volume. There was no significant difference between patients and controls in whole-brain and right hippocampal volume. Decreased AKT ratio was associated with reduced hippocampal volume. There was no significant relationship between ERK ratio and brain structure.

Conclusion: Activation of the AKT system is specifically associated with hippocampal volume in firstepisode schizophrenia, which provides further evidence for the pivotal role of this messenger system in the pathophysiology of psychotic disorders.
\end{abstract}

(c) 2011 Elsevier Ltd. All rights reserved.

\section{Introduction}

The complexity of the pathophysiology of schizophrenia and related psychotic disorders raises important questions regarding master regulator mechanisms integrating diverse molecular factors. The phosphoinositide $3^{\prime}$-kinase (PI3K) - protein kinase B (AKT1) - glycogen synthase kinase (GSK)-3 $\beta$ intracellular signaling system is modulated by dopamine, serotonin, glutamate, neuregulin 1 (NRG1), dysbindin, and Disrupted in Schizophrenia-1 (DISC-1), representing a reliable target for antipsychotics and mood stabilizers (Kalkman, 2006; Bellon, 2007; Freyberg et al., 2010; Karam et al., 2010; Kvajo et al., 2010; Beaulieu, 2011). Recently, we found that a genome-wide association identified

\footnotetext{
* Corresponding author. University of Szeged, Faculty of Medicine, Department of Physiology, Szeged, Hungary. Tel.: +36 204483530.

E-mail addresses: szkeri2000@yahoo.com, keri.szabolcs.gyula@med.u-szeged. hu (S. Kéri).
}

psychosis risk variant of the CACNA1C gene, encoding the alpha subunit of the L-type voltage-dependent calcium channel, also affects the AKT system (Balog et al., 2010).

Regarding structural brain alterations, the hippocampus may play a critical role in schizophrenia because multiple pathophysiological factors converge on this brain structure (Freedman and Goldowitz, 2010; Heckers and Conradi, 2010; Tamminga et al., 2010; Lodge and Grace, 2011). Tan et al. (2011) reported that a schizophrenia-related polymorphism of the $A K T$ gene is linked to hippocampal structure and function, with an epistasis with functional polymorphisms in Brain Derived Neurotrophic Factor (BDNF) and Catechol-O-Methyltransferase (COMT) genes. Lower serum BDNF concentration correlates with decreased hippocampal volume in first-episode schizophrenia (Rizos et al., 2011). Finally, poor sensory gating, a putative marker of hippocampal dysfunction, is associated with decreased NRG1-induced AKT activation in lymphoblasts of firstepisode patients with schizophrenia (Kéri et al., 2010).

NRG1, a candidate susceptibility factor for psychotic disorders, acts on Epidermal Growth Factor receptors (ErbBs) and plays an 
essential role in neurodevelopment and synaptic plasticity (Harrison and Law, 2006; Mei and Xiong, 2008; Schmitt et al., 2008; Buonanno, 2010). Although genome-wide association studies did not confirm its association with psychotic disorders (O'Donovan et al., 2008; The Schizophrenia Psychiatric Genome-Wide Association Study Consortium, 2011), gene expression profiles of neurons derived from reprogrammed fibroblasts of schizophrenia patients into human pluripotent stem cells revealed abnormal patterns of NRG1 expression (Brennand et al., 2011). Sei et al. (2007) investigated NRG1-induced migration of lymphoblasts and demonstrated decreased migration in patients with schizophrenia relative to controls. From a genetic point of view, the magnitude of NRG1induced lymphoblast migration was associated with polymorphisms of the NRG1, COMT, and AKT genes (Sei et al., 2007, 2010), and NRG1 risk polymorphisms are associated with decreased hippocampal volume (Gruber et al., 2008).

One of the master regulator mechanisms responsible for impaired lymphoblast migration may be decreased AKT phosphorylation in patients with schizophrenia (Sei et al., 2007). Decreased AKT phosphorylation in lymphoblasts of patients is also related to diminished suppression of the P50 event-related potential (Kéri et al., 2010), impaired habituation of autonomic arousal, and delusional ideations in non-clinical individuals (Kéri et al., 2011). However, it is not known whether AKT activation is linked to structural alterations of the hippocampus, which is implicated in various intermediate phenotypes and clinical symptoms of schizophrenia (Freedman and Goldowitz, 2010; Heckers and Conradi, 2010; Tamminga et al., 2010; Lodge and Grace, 2011). To answer this question, we measured NRG1induced activation of intracellular messenger systems in peripheral lymphoblasts of schizophrenia patients and controls, and calculated correlations with volumes of brain structures. In addition to the AKT system, we also investigated NRG1-induced phosphorylation of ERK (extracellular signal-regulated kinase) in order to test the specificity of potential relationship with brain structure (Sei et al., 2007).

\section{Methods}

\subsection{Participants}

Twenty patients with first-episode schizophrenia and twenty healthy controls participated in the study. The patients had a first episode of schizophrenia and did not receive psychotropic medications. The control participants were hospital employees, their spouses, and acquaintances. All participants received the Structured Clinical Interview for DSM-IV axis I disorders (SCID-CV) (First et al., 1996). The control participants had no Axis I psychiatric disorders and had a negative family history for psychotic disorders. Detailed medical records were available from each patient, including reports from parents and other relatives. Exclusion criteria included neurological disorders, head injury, history of psychoactive substance dependence, and positive toxicological screening. For the assessment of clinical symptoms, we used the Positive and Negative Syndrome Scale (PANSS) (Kay et al., 1987). Table 1 presents the clinical and demographic characteristics. The study was carried out in accordance with the Declaration of Helsinki and was approved by the local ethics board. All participants were able to give written informed consent.

\subsection{AKT and ERK phosphorylation}

We used standard methods reported previously (Sei et al., 2007, 2010; Kéri et al., 2009, 2010, 2011). B lymphocytes in the mononuclear cell preparation were transformed by Epstein-Barr virus. The lymphoblasts were stimulated with NRG1A for $30 \mathrm{~min}$. We performed Western blots to analyze proteins isolated from the cells. To quantify phosphorylation, the immunoblots were stained with antibodies specific to phosphorylated ERK (Tyr180; 42, $44 \mathrm{kDa}$ ) or AKT (Ser473; $60 \mathrm{kDa}$ ) and were then stripped and probed with antibodies to total ERK or AKT. The primary antibodies were detected using horseradish peroxidase-conjugated anti-rabbit IgG antibodies. We measured the relative optical density using the National Institute of Health Image software. The dependent measure was the ratio of phosphorylated AKT (pAKT) or phosphorylated ERK (pERK) and total AKT or total ERK at baseline (without NRG1 stimulation) and after NRG1 stimulation.

\subsection{Structural brain imaging}

Each participant underwent a head magnetic resonance (MR) imaging (Philips Intera $1.5 \mathrm{~T}$ scanner; T1-weighted 3D/FFE gradient-echo sequence, TR/TE $14.3 / 3.3 \mathrm{~ms}$, flip angle $30^{\circ}$, field of view 240 , matrix $256 \times 256$, slab thickness $2 \mathrm{~mm}, 1.5 \mathrm{~mm}$ spacing, 124-130 partitions, inplane resolution: $0.94 \times 0.94 \mathrm{~mm}$ ). We used the validated standard FreeSurfer image analysis pipeline (Martinos Center for Biomedical Imaging, Boston, MA, USA; http://surfer.nmr mgh.harvard.edu/). Image processing included the removal of nonbrain tissue with a hybrid watershed/surface deformation procedure, automated Talairach transformation, and segmentation of the subcortical white and gray matter (Fischl et al., 2004; Segonne et al., 2004). We measured whole-brain, right, and left hippocampal volume.

\subsection{Data analysis}

We used STATISTICA 9 (StatSoft, Inc., Tulsa) software for data analysis. The normality of data distribution and the homogeneity of variance were checked using Kolmogorov-Smirnov and Levene's tests, respectively. We conducted repeated measures analyses of variance (ANOVAs), followed by Scheffé's post hoc tests, or twotailed $t$ tests to compare patients and controls. We calculated

Table 1

Clinical and demographic description of the participants.

\begin{tabular}{|c|c|c|c|c|c|c|}
\hline & \multicolumn{3}{|c|}{ Controls $(n=20) 13$ male/7 female } & \multicolumn{3}{|c|}{ Schizophrenia $(n=20) 13$ male/7 female } \\
\hline & Mean & SD & range & Mean & SD & range \\
\hline Age (years) & 24.7 & 6.2 & $19-30$ & 24.3 & 5.4 & $19-29$ \\
\hline Education (years) & 11.5 & 4.5 & $8-17$ & 11.0 & 3.6 & $8-17$ \\
\hline Duration of untreated psychosis (months) & - & & & 10.3 & 4.8 & $7-15$ \\
\hline PANSS positive & - & & & 18.6 & 5.1 & $14-25$ \\
\hline PANSS negative & - & & & 17.4 & 5.3 & $15-27$ \\
\hline PANSS general & - & & & 35.4 & 6.1 & $30-43$ \\
\hline
\end{tabular}

PANSS - Positive and Negative Syndrome Scale. Controls and patients did not differ in age and education $(p>0.5, t$ test) and were matched for gender ratio. 



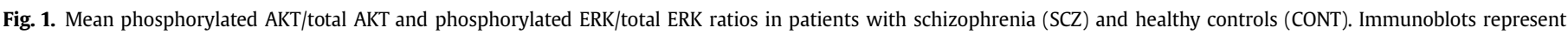
samples from the NRG1-induced condition. Error bars indicate $95 \%$ confidence intervals. ${ }^{*} p<0.001$, Scheffé's test.

Pearson's product moment correlation coefficients and used linear regression analysis to determine associations between AKT/ERK ratios and brain volume. The level of significance was set at $\alpha<0.05$

\section{Results}

\subsection{AKT and ERK ratios}

Fig. 1 depicts the AKT and ERK ratios (phosphorylated protein/ total protein) at baseline and after NRG1 stimulation. We conducted an ANOVA in which group was the between-subjects factor and ratio (AKT vs. ERK), and condition (baseline vs. NRG1 stimulation) were the within-subjects factors. The ANOVA revealed significant main effects of group $(F(1,38)=24.46, p<0.001)$, ratio $(F(1,38)=26.37, p<0.001)$, and condition $(F(1,38)=30.16$, $p<0.001)$. There were significant two-way interactions between group and ratio $(F(1,38)=6.34, p<0.05)$, and group and condition $(F(1,38)=12.71, p<0.01)$. The three-way interaction among group, ratio, and condition was also significant $(F(1,38)=19.71, p<0.001)$.

Scheffé's tests revealed a single condition in which the values were significantly lower in patients relative to controls: the NRG1induced AKT ratio $(p<0.001)$ (Fig. 1$)$.

\subsection{Brain volumes}

Table 2 presents the results from brain volume measurements. Patients displayed significantly reduced left hippocampal volume relative to controls, whereas in the case of right hippocampus and whole-brain volume the difference did not reach the level of statistical significance.

\subsection{Correlations between molecular markers and brain structure}

Table 3 presents the correlation coefficients between AKT and ERK ratios and brain volumes in patients with schizophrenia. Left and right hippocampal volumes correlated with NRG1-induced AKT activation (Fig. 2). In the control group, the correlations were not significant, but in the case of hippocampus there was a positive relationship similar to that observed in patients with schizophrenia $(\mathrm{r}[$ left $]=0.31, p=0.3 ; \mathrm{r}[$ right $]=0.28, p=0.4)$.

We further examined the relationship between hippocampal volume and NRG1-induced AKT activation in schizophrenia patients using linear regression analysis with age, education, gender, symptoms (PANSS scores), and whole-brain volume. For both left and right hippocampi, only AKT activation was a significant predictor (left: $b^{*}=0.54, t(13)=2.23, p<0.05$; right: $b^{*}=0.62, t(13)=2.64$, $p<0.05)$.

\section{Discussion}

The results of the present study revealed that diminished NRG1induced AKT phosphorylation in peripheral lymphoblasts is associated with smaller hippocampal but not whole-brain volume in schizophrenia. In contrast, ERK phosphorylation was not related to brain structure and did not show abnormality in schizophrenia. ERKs, also called mitogen-activated protein kinases (MAPKs), are ubiquitous intracellular signaling molecules modulated by various growth factors and neurotransmitters, regulating cell division, differentiation, and metabolism (Keshet and Seger, 2010). Given that this system was intact in schizophrenia, our results are against the possibility of a generalized dysfunction of widespread intracellular messenger systems. Furthermore, we investigated first-

Table 2

Hippocampal and whole-brain volumes.

\begin{tabular}{|c|c|c|c|c|c|c|}
\hline & Controls $(n=20)$ & Schizophrenia $(n=20)$ & $t$ & $p$ & $d$ & Decrease in patients \\
\hline Left hippocampus $\left(\mathrm{mm}^{3}\right)$ & $3566.0(292.1)$ & $3354.5(227.6)$ & 2.55 & 0.01 & 0.75 & $6 \%$ \\
\hline Right hippocampus $\left(\mathrm{mm}^{3}\right)$ & $3627.5(285.1)$ & $3480.5(227.7)$ & 1.80 & 0.08 & 0.55 & $4 \%$ \\
\hline Whole-brain $\left(\mathrm{cm}^{3}\right)$ & $1325.0(119.4)$ & $1295.1(104.2)$ & 0.86 & 0.39 & 0.27 & $2 \%$ \\
\hline
\end{tabular}

Data are mean $(\mathrm{SD})$. Results from two-tailed $t$ tests $(\mathrm{df}=38)$ and effect size values (Cohen's $d$ ) are shown. 
Table 3

Correlations among AKT/ERK ratios and brain structure in patients with schizophrenia $(n=20)$.

\begin{tabular}{llcc}
\hline & $\begin{array}{l}\text { Left } \\
\text { hippocampus }\end{array}$ & $\begin{array}{l}\text { Right } \\
\text { hippocampus }\end{array}$ & $\begin{array}{l}\text { Whole-brain } \\
\text { volume }\end{array}$ \\
\hline AKT baseline & 0.17 & 0.27 & 0.16 \\
AKT NRG1-induced & $0.52^{*}$ & $0.61^{*}$ & -0.01 \\
ERK baseline & 0.12 & -0.09 & -0.11 \\
ERK NRG1-induced & 0.18 & 0.02 & 0.07 \\
\hline
\end{tabular}

The table depicts Pearson's product moment correlation coefficients. ${ }^{*} p<0.05$.

episode patients who did not receive psychotropic medications, and therefore the confounding effect of drugs and adversity accompanying disease chronicity can be excluded. Although impaired AKT activation seems to be similar in first-episode and chronic patients (Kéri et al., 2009, 2010), longitudinal studies are warranted to explore the effects of medications and illness course.

Mechanisms associated with neurodevelopment and neuroplasticity could explain why impaired NRG1-induced AKT activation might affect hippocampal structure. According to Bellon (2007), NRG1 and AKT are important in neurite formation, a crucial stage in development, which may be impaired in schizophrenia. NRG1 is a key regulator in the development of GABA-ergic neuronal circuits (Fazzari et al., 2010; Ting et al., 2011). There is increasing evidence that NRG1 in the peripheral circulation influences postnatal neurodevelopment and neuroplasticity, and these processes are related to GABA-ergic interneurons and their glutamatergic innervation (Abe et al., 2011). Rösler et al. (2011) showed that the extracellular domain of peripheral NRG1 passes the blood-brain barrier and activates receptors in the brain. In adult mice, peripherally administered NRG1 enhanced neurogenesis in the hippocampus (Mahar et al., 2011). Our results may provide a link between basic and clinical neuroscience, motivating future studies to assess the cellular correlates of brain volume loss in schizophrenia and its relationship with specific cell types in the hippocampus and prefrontal cortex (Lewis and González-Burgos, 2008; Lisman et al., 2008).

It is important to emphasize that our results are indirect, as there is no evidence that NRG1-induced AKT phosphorylation is the same in neurons and peripheral lymphoblasts. However,Balu et al. (2010) examined postmortem tissue from patients with schizophrenia and showed decreased phosphorylated AKT levels in the dentate gyrus, the neurogenic zone of the hippocampus. Nevertheless, correlation data between peripheral markers and brain structures must be treated with caution and need to be replicated and extended in studies allowing conclusions based on causal relationships.

It is also not fully clarified whether NRG1-induced AKT activation is illness state-dependent or reflects a trait affecting early circuit formation in the hippocampus before the appearance of psychotic symptoms. In a small group of monozygotic twins discordant for schizophrenia, we found abnormal AKT activation only in affected twins but not in healthy co-twins, although the healthy co-twins' activation level was lower than that of the unrelated controls (Seres et al., 2010). If AKT activation is a state-marker, it may be related to other aspects of neuroplasticity, such as dendritic arborization, cell size, and probably cell division. Chakos et al. (2005) demonstrated that decreased hippocampal volume is not merely a stable trait marker of schizophrenia. Specifically, they showed that in an early illness group second-generation antipsychotics were associated with larger hippocampal volumes (Chakos et al., 2005). The exact cellular mechanism of this effect is not clear, but the AKT system may be a candidate mediating the effects of antipsychotics on neuroplasticity (Freyberg et al., 2010).

Decreased hippocampal volume is one of the most frequently reported structural brain abnormality in schizophrenia (Nelson et al., 1998; Wright et al., 2000; Honea et al., 2005; Steen et al., 2006; Vita et al., 2006; Adriano et al., 2011) although its disease specificity is low, and its magnitude is moderate and variable across studies (Geuze et al., 2005). For example, Steen et al. (2006) reported hippocampal volume deficit of $8 \%$ in first-episode patients, whereas Nelson et al. (1998) found much smaller volume loss in chronic patients (4\%), which can be considered as a paradoxical finding. It is notable, however, that $4 \%$ volume difference is within the range of "machine drift", which is due to various factors including voxel misclassification, partial volume effect, and head tilt (Steen et al., 2006). There is meta-analytic evidence for hippocampal volume reduction in first-episode schizophrenia (Steen et al., 2006; Vita

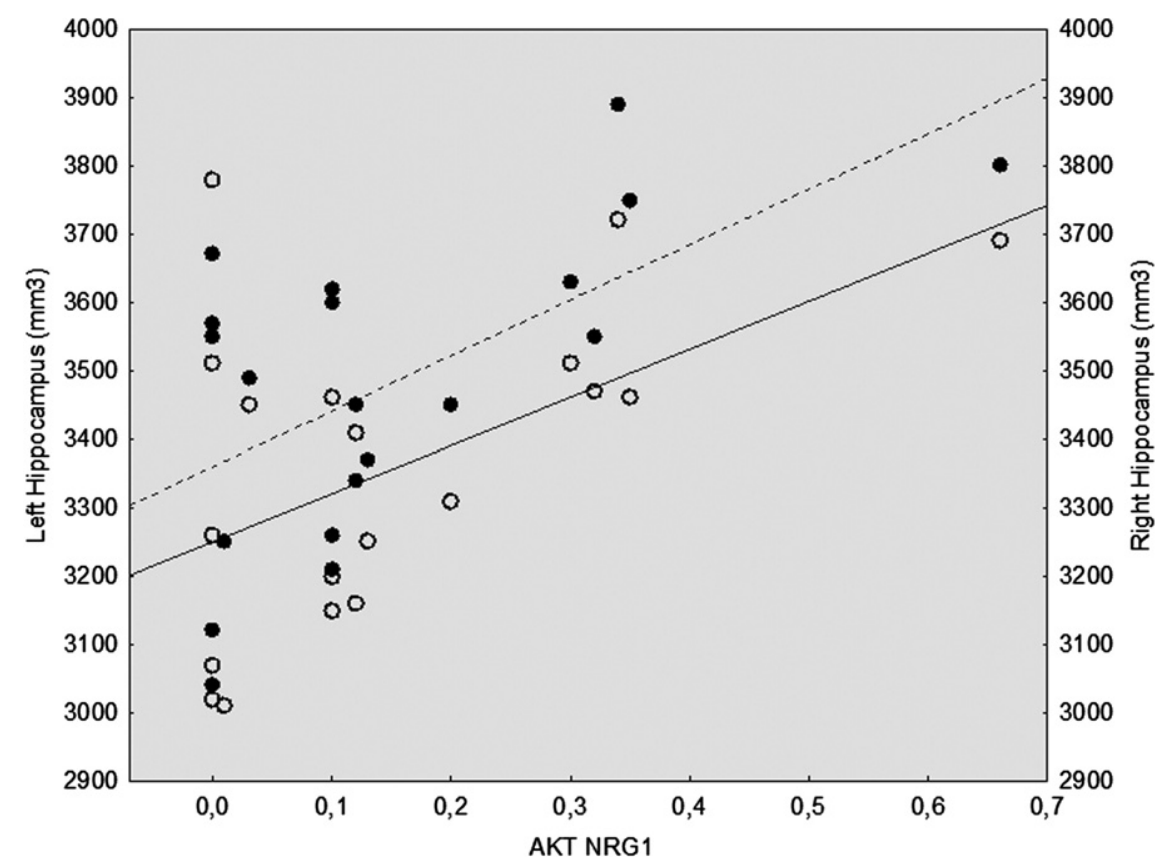

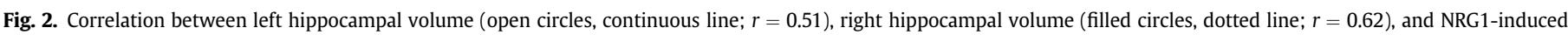
phosphorylated AKT/total AKT ratio. 
et al., 2006; Adriano et al., 2011), and others identified volume loss in the uncus/amygdala complex (Ellison-Wright et al., 2008). Recent studies found hippocampal volume reduction not only in firstepisode schizophrenia, but also in "high-risk" individuals with prodromal symptoms (Ebdrup et al., 2010; Witthaus et al., 2010; for a review of "high-risk" studies, see Fusar-Poli et al., 2011), although others suggested that hippocampal volume loss is associated with the first psychotic episode (Buehlmann et al., 2010).

Despite the fact that we demonstrated a specific molecular correlate of hippocampal volume reduction, it is important to note that non-specific factors may also account for such deficits. Mondelli et al. (2010) reported that higher cortisol levels, a marker of general stress response, are associated with smaller left hippocampus in first-episode psychosis. The confounding effect of such non-specific factors should be investigated by future studies.

The most important limitations of the present study are small sample size and MR scanning at 1.5 T. Slab thickness and spacing between slabs do not allow a high-resolution mapping and a proper investigation of hippocampal subregions. Moreover, it is essential to clarify how antipsychotics modulate the AKT system in firstepisode patients, and how it relates to longitudinal structural changes of the hippocampal formation and other brain structures.

In conclusion, peripheral markers of impaired intracellular signaling processes may be putative markers of schizophrenia. These molecular alterations specifically correlate with structural brain changes. Our data indicate that the relationship between NRG1-induced AKT phosphorylation and hippocampal structure and function (Kéri et al., 2010) may be especially relevant.

\section{Role of funding source}

The Hungarian Research Fund (OTKA NF72488) provided support for this study. The sponsor had no further role in study design, in the collection, analysis and interpretation of data, in the writing of the report, and the decision to submit the paper.

\section{Contributors}

Dr. Kéri and Dr. Kelemen designed the study, Mr. Szamosi collected the data, performed the initial analysis, and wrote the first draft of the paper, dr. Kéri completed the data analysis, and all authors contributed to the final version of the manuscript. All authors approved its publication.

\section{Conflict of interest statement}

The authors declare no competing interest.

\section{Acknowledgments}

We thank Ferenc Halász, Zsolt Balog, and Gabriella Havas for technical assistance.

\section{References}

Abe Y, Namba H, Kato T, Iwakura Y, Nawa H. Neuregulin-1 signals from the periphery regulate AMPA receptor sensitivity and expression in GABAergic interneurons in developing neocortex. Journal of Neuroscience 2011;31: 5699-709.

Adriano F, Caltagirone C, Spalletta G. Hippocampal volume reduction in firstepisode and chronic schizophrenia: a review and meta-analysis. Neuroscientist; 2011 Apr 29. doi: 10.1177/1073858410395147.

Balog Z, Kiss I, Kéri S. CACNA1C risk allele for psychotic disorders is related to the activation of the AKT-pathway. American Journal of Psychiatry 2010;167: 1276-7.

Balu DT, Carlson GC, Talbot K, Kazi H, Hill-Smith TE, Easton RM, et al. Akt1 deficiency in schizophrenia and impairment of hippocampal plasticity and function. Hippocampus; 2010 Nov 3. doi:10.1002/hipo.20887.
Beaulieu JM. A role for Akt and glycogen synthase kinase-3 as integrators of dopamine and serotonin neurotransmission in mental health. Journal of Psychiatry and Neuroscience 2011;36:110011. doi: 10.1503/jpn.110011.

Bellon A. New genes associated with schizophrenia in neurite formation: a review of cell culture experiments. Molecular Psychiatry 2007;12:620-9.

Brennand KJ, Simone A, Jou J, Gelboin-Burkhart C, Tran N, Sangar S, et al. Modelling schizophrenia using human induced pluripotent stem cells. Nature; 2011 Oct 19. doi: 10.1038/nature10603.

Buehlmann E, Berger GE, Aston J, Gschwandtner U, Pflueger MO, Borgwardt SJ, et al. Hippocampus abnormalities in at risk mental states for psychosis? A crosssectional high resolution region of interest magnetic resonance imaging study. Journal of Psychiatric Research 2010;44:447-53.

Buonanno A. The neuregulin signaling pathway and schizophrenia: from genes to synapses and neural circuits. Brain Research Bulletin 2010;83:122-31.

Chakos MH, Schobel SA, Gu H, Gerig G, Bradford D, Charles C, et al. Duration of illness and treatment effects on hippocampal volume in male patients with schizophrenia. British Journal of Psychiatry 2005;186:26-31.

Ebdrup BH, Glenthøj B, Rasmussen H, Aggernaes B, Langkilde AR, Paulson OB, et al. Hippocampal and caudate volume reductions in antipsychotic-naive firstepisode schizophrenia. Journal of Psychiatry and Neuroscience 2010;35: 95-104.

Ellison-Wright I, Glahn DC, Laird AR, Thelen SM, Bullmore E. The anatomy of firstepisode and chronic schizophrenia: an anatomical likelihood estimation metaanalysis. American Journal of Psychiatry 2008;165:1015-23.

Fazzari P, Paternain AV, Valiente M, Pla R, Luján R, Lloyd K, et al. Control of cortical GABA circuitry development by Nrg1 and ErbB4 signalling. Nature 2010;464: 1376-80.

First MB, Spitzer RL, Gibbon M, Williams JBW. Structured Clinical Interview for DSM-IV Axis I Disorders, Clinician Version (SCID-CV). Washington, DC: American Psychiatric Press; 1996.

Fischl B, Salat DH, van der Kouwe AJ, Makris N, Ségonne F, Quinn BT, et al. Sequence-independent segmentation of magnetic resonance images. Neuroimage 2004;23(Suppl. 1):S69-84.

Freedman R, Goldowitz D. Studies on the hippocampal formation: from basic development to clinical applications: studies on schizophrenia. Progress in Neurobiology 2010;90:263-75.

Freyberg Z, Ferrando SJ, Javitch JA. Roles of the Akt/GSK-3 and Wnt signaling pathways in schizophrenia and antipsychotic drug action. American Journal of Psychiatry 2010;167:388-96.

Fusar-Poli P, Borgwardt S, Crescini A, Deste G, Kempton MJ, Lawrie S, et al. Neuroanatomy of vulnerability to psychosis: a voxel-based meta-analysis. Neuroscience and Biobehavioral Reviews 2011;35:1175-85.

Geuze E, Vermetten E, Bremner JD. MR-based in vivo hippocampal volumetrics: 2. Findings in neuropsychiatric disorders. Molecular Psychiatry 2005;10: $160-84$.

Gruber O, Falkai P, Schneider-Axmann T, Schwab SG, Wagner M, Maier W. Neuregulin-1 haplotype $\mathrm{HAP}$ (ICE) is associated with lower hippocampal volumes in schizophrenic patients and in non-affected family members. Journal of Psychiatric Research 2008;43:1-6.

Harrison PJ, Law AJ. Neuregulin 1 and schizophrenia: genetics, gene expression, and neurobiology. Biological Psychiatry 2006;60:132-40.

Heckers S, Konradi C. Hippocampal pathology in schizophrenia. Current Topics in Behavioural Neurosciences 2010;4:529-53.

Honea R, Crow TJ, Passingham D, Mackay CE. Regional deficits in brain volume in schizophrenia: a meta-analysis of voxel-based morphometry studies. American Journal of Psychiatry 2005;162:2233-45.

Kalkman HO. The role of the phosphatidylinositide 3-kinase-protein kinase B pathway in schizophrenia. Pharmacology \& Therapeutics 2006;110:117-34.

Karam CS, Ballon JS, Bivens NM, Freyberg Z, Girgis RR, Lizardi-Ortiz JE, et al. Signaling pathways in schizophrenia: emerging targets and therapeutic strategies. Trends in Pharmacological Sciences 2010;31:381-90.

Kay SR, Fiszbein A, Opler LA. The positive and negative syndrome scale (PANSS) for schizophrenia. Schizophrenia Bulletin 1987;13:261-76.

Kéri S, Seres I, Kelemen O, Benedek G. Neuregulin 1-stimulated phosphorylation of AKT in psychotic disorders and its relationship with neurocognitive functions. Neurochemistry International 2009;55:606-9.

Kéri S, Beniczky S, Kelemen O. Suppression of the P50 evoked response and neuregulin 1-induced AKT phosphorylation in first-episode schizophrenia. American Journal of Psychiatry 2010;167:444-50.

Kéri S, Seres I, Kelemen O, Benedek G. The relationship among neuregulin 1stimulated phosphorylation of AKT, psychosis proneness, and habituation of arousal in nonclinical individuals. Schizophrenia Bulletin 2011;37:141-7.

Keshet Y, Seger R. The MAP kinase signaling cascades: a system of hundreds of components regulates a diverse array of physiological functions. Methods in Molecular Biology 2010;661:3-38.

Kvajo M, McKellar H, Gogos JA. Molecules, signaling, and schizophrenia. Current Topics in Behavioural Neurosciences 2010;4:629-56.

Lewis DA, González-Burgos G. Neuroplasticity of neocortical circuits in schizophrenia. Neuropsychopharmacology 2008;33:141-65.

Lisman JE, Coyle JT, Green RW, Javitt DC, Benes FM, Heckers S, et al. Circuit-based framework for understanding neurotransmitter and risk gene interactions in schizophrenia. Trends in Neurosciences 2008;31:234-42.

Lodge DJ, Grace AA. Developmental pathology, dopamine, stress and schizophrenia. International Journal of Developmental Neurosciences 2011;29:207-13. 
284

A. Szamosi et al. / Journal of Psychiatric Research 46 (2012) 279-284

Mahar I, Tan S, Davoli MA, Dominguez-Lopez S, Qiang C, Rachalski A, et al. Subchronic peripheral neuregulin-1 increases ventral hippocampal neurogenesis and induces antidepressant-like effects. PLo One 2011;6:e26610.

Mri L, Xiong WC. Neuregulin 1 in neural development, synaptic plasticity and schizophrenia. Nature Review Neurosciences 2008;9:437-52.

Mondelli V, Pariante CM, Navari S, Ans M, D'Albenzio A, Di Fort M, et al. Higher cortisol levels are associated with smaller left hippocampal volume in firstepisode psychosis. Schizophrenia Research 2010;119:75-8.

Nelson MD, Saykin AJ, Flashman LA, Riordan HJ. Hippocampal volume reduction in schizophrenia as assessed by magnetic resonance imaging: a meta-analytic study. Archives of General Psychiatry 1998;55:433-40.

O'Donovan MC, Braddock N, Norton N, Williams H, Peirce T, Moskvina V, et al. Identification of loci associated with schizophrenia by genome-wide associaion and follow-up. Nature Genetics 2008;40:1053-5.

Rizos EN, Papathanasiou M, Michalopoulou PG, Mazioti A, Douzenis A, Kastania A, et al. Association of serum BDNF levels with hippocampal volumes in first psychotic episode drug-naive schizophrenic patients. Schizophrenia Research 2011;129:201-4

Rösler TW, Depboylu C, Arias-Carrión O, Wozny W, Carlsson T, Höllerhage M, et al. Biodistribution and brain permeability of the extracellular domain of newregulin-1- $\beta 1$. Neuropharmacology 2011;61:1413-8.

Schmitt A, Parlapani E, Gruber O, Wobrock T, Falkai P. Impact of neuregulin-1 on the pathophysiology of schizophrenia in human post-mortem studies. European Archives of Psychiatry and Clinical Neuroscience 2008;258:35-9.

Segonne F, Dale AM, Bursa E, Glessner M, Salas D, Hahn HK, et al. A hybrid approach to the skull stripping problem in MRI. Neuroimage 2004;22:1060-75.

See Y, Ren-Patterson R, Li Z, Tunbridge EM, Egan MF, Kolachana BS, et al. Nuregulin1-induced cell migration is impaired in schizophrenia: association with neuregulin1 and catechol-o-methyltransferase gene polymorphisms. Molecular Psychiatry 2007;12:946-57.
See Y, Li Z, Song J, Ren-Patterson R, Tunbridge EM, Iizuka Y, et al. Epistatic and functional interactions of catechol-o-methyltransferase (COMT) and AKT1 on neuregulin1-ErbB signaling in cell models. PLo One 2010;5:e10789.

Sere I, Kelemen O, Benedek G, Kéri S. Neuregulin 1-induced AKT phosphorylation in monozygotic twins discordant for schizophrenia. Neurochemistry Internetonal 2010;56:906-10.

Steen RG, Mull C, McClure R, Homer RM, Lieberman JA. Brain volume in firstepisode schizophrenia: systematic review and meta-analysis of magnetic resonance imaging studies. British Journal of Psychiatry 2006;188:510-8.

Tamminga CA, Stan AD, Wagner AD. The hippocampal formation in schizophrenia. American Journal of Psychiatry 2010;167:1178-93.

Tan HY, Chen AG, Chen Q Browne LB, Verchinski B, Kolachana B, et al. Epistatic interactions of AKT1 on human medial temporal lobe biology and pharmacogemetic implications. Molecular Psychiatry; 2011 Jul 26. do: 10.1038/mp.2011.91.

The Schizophrenia Psychiatric Genome-Wide Association Study (GWAS) Consortum, Ripke S, Sanders AR, Kendler KS, Levinson DF, Sklar P, Holman PA, et al. Genome-wide association study identifies five new schizophrenia loci. Nature Genetics 2011;43:969-76.

Ting AK, Chen Y, Wen L, Yin DM, She C, Tao Y, et al. Neuregulin 1 promotes excitatory synapse development and function in GABAergic interneurons. Journal of Neuroscience 2011;31:15-25.

Vita A, De Peri L, Silenzi C, Dieci M. Brain morphology in first-episode schizophrenia: a meta-analysis of quantitative magnetic resonance imaging studies. Schizophrenia Research 2006;82:75-88.

Witthaus H, Mended U, Brüne M, Ozgürdal S, Bohner G, Gudlowski Y, et al. Hippocampal subdivision and amygdalas volumes in patients in an at-risk mental state for schizophrenia. Journal of Psychiatry and Neuroscience 2010;35:33-40.

Wright IC, Rabe-Hesketh S, Woodruff PW, David AS, Murray RM, Bullmore ET. Metaanalysis of regional brain volumes in schizophrenia. American Journal of Psychiatry 2000;157:16-25. 